\title{
Barcoding type specimens helps to identify synonyms and an unnamed new species in Eumunida Smith, 1883 (Decapoda : Eumunididae)
}

\author{
Nicolas Puillandre ${ }^{\mathrm{A}, \mathrm{E}}$, Enrique Macpherson ${ }^{\mathrm{B}}$, Josie Lambourdière ${ }^{\mathrm{C}}$, Corinne Cruaud ${ }^{\mathrm{D}}$, \\ Marie-Catherine Boisselier-Dubayle ${ }^{\mathrm{A}, \mathrm{B}, \mathrm{C}}$ and $S \rightleftharpoons$ Samadi ${ }^{\mathrm{A}, \mathrm{B}, \mathrm{C}}$ \\ A'Systématique, Adaptation et Evolution', UMR 7138 UPMC-IRD-MNHN-CNRS (UR IRD 148), \\ Muséum national d'Histoire naturelle, Département Systématique et Evolution, CP 26, \\ 57 Rue Cuvier, F-75231 Paris Cedex 05, France. \\ ${ }^{B}$ Centro de Estudios Avanzados de Blanes (CEAB-CSIC), C. acc. Cala SantFrancesc 14, 17300 Blanes, Girona, Spain. \\ ${ }^{C}$ Service de systématique moléculaire (CNRS-MNHN, UMS2700), Muséum national d'Histoire naturelle, \\ Département Systématique et Evolution, CP 26, 57 Rue Cuvier, F-75231 Paris Cedex 05, France. \\ ${ }^{D}$ GENOSCOPE, Centre National de Séquençage, 2 rue Gaston Crémieux, CP 5706, 91057 Evry Cedex France. \\ ${ }^{\mathrm{E}}$ Corresponding author. Email: puillandre@mnhn.fr
}

\begin{abstract}
The primary purpose of DNA-barcoding projects is to generate an efficient expertise and identification tool. This is an important challenge to the taxonomy of the 21 st century, as the demand increases and the expert capacity does not. However, identifying specimens using DNA-barcodes requires a preliminary analysis to relate molecular clusters to available scientific names. Through a case study of the genus Eumunida (Decapoda: Eumunididae), we illustrate how naming molecule-based units, and thus providing an accurate DNA-based identification tool, is facilitated by sequencing type specimens. Using both morphological and unlinked molecular markers (COI and 28S genes), we analysed 230 specimens from 12 geographic areas, covering two-thirds of the known diversity of the genus, including type specimens of 13 species. Most hypotheses of species delimitation are validated, as they correspond to molecular units linked to only one taxonomic name (and vice versa). However, a putative cryptic species is also revealed and three entities previously named as distinct species may in fact belong to a single one, and thus need to be synonymised. Our analyses, which integrate the current naming rules, enhance the $\alpha$-taxonomy of the genus and provide an effective identification tool based on DNA-barcodes. They illustrate the ability of DNA-barcodes, especially when type specimens are included, to pinpoint where a taxonomic revision is needed.
\end{abstract}

Received 25 May 2011, accepted 9 November 2011, published online dd mmm yyyy

\section{Introduction}

When describing a new species, the taxonomists provide a species name and designate one (or several) type specimens to which this name is permanently attached. A species name allows us to designate a testable species hypothesis, and the type specimens provide the link with the name of this hypothesis. Designating species hypotheses by species names allows anyone to associate newly examined specimens to already proposed species hypotheses. However, proposing species hypotheses, species names and species identifications are three distinct tasks that should not be confused (Dayrat 2006). They can be distinguished as follows: (1) the scientific task consists of proposing hypotheses about species boundaries, based on the comparison of characters or on biological criteria; (2) the naming task deals with assigning names to such species hypotheses; and (3) the identification task is to identify specimens in the light of already named species hypotheses.
Within this methodological framework, the primary purpose of DNA barcoding projects is not to produce new taxonomic hypotheses and to name them - Tasks 1 and 2 - but to facilitate taxonomic identification - Task 3 - by developing a global standard for the identification of biological species based on molecular data (Hebert and Gregory 2005; Schindel and Miller 2005). However, identifying specimens using only their barcode sequences requires a database that includes the sequences and the corresponding specimen data, authoritatively identified using morphological characters. Furthermore, a prior analysis of the molecular diversity of the groups is necessary to confirm (or reject) that DNA barcodes may be used as a diagnostic character for the species at hand, i.e. that intraspecific and interspecific genetic distances are separated by a 'barcode gap'. In that way, the identification of new specimens using such a DNA library would follow the opinion of the taxonomist that has identified the specimens of the DNA barcode library. Here, two problems 
need to be addressed. First, a link between DNA-based species hypotheses and already available morphological species hypotheses (and thus species names) needs to be assessed. For example, in the study of Smith et al. (2007), it was not possible to ascertain the link between genetic clusters and available names with full confidence because no DNA barcode was obtained for the holotype; this uncertainty in the assignation of species names to species hypotheses was indicated by indicating the scientific names in quotation marks. Second, one important by-product of DNA barcoding as an identification tool for taxonomy is the detection of specimens that cannot be attributed to any available species hypothesis, and for which a new hypothesis - and thus a new name - may be proposed (e.g. Padial and De La Riva 2007). Once again, the attribution of available species names to genetic clusters is critical to clearly highlight genetic clusters that would deserve a new species name. Thus, because DNA barcodes can be used both to attribute species names to a given specimen and to flag genetic clusters for which no name is available, we should clarify how names are - or should be - given to species hypotheses. This can be achieved by the sequencing of type specimens.

Using a case study of the genus Eumunida Smith, 1883 (Decapoda: Chirostyloidea : Eumunididae), we illustrate here the difficulties of this naming task, in the context of the development of DNA barcodes as an identification tool. We selected this genus because most species have been described recently and the conservation of name-bearing specimens in the collections allows us to access molecular characters. Many species were described using material that has been preserved in $70 \%$ ethanol, the samples are housed in the collection of the Museum National d'Histoire Naturelle in Paris, having been collected over a quarter of a century's exploration in the southwest Pacific (Bouchet et al. 2008). In this case study we integrate the three tasks of taxonomy. Our specific aims are thus: (1) to test the robustness of recognised species hypotheses and, if needed, to propose new ones; and (2) to name the revised set of species hypotheses. This way, the efficiency of DNA barcodes as an identification key will also be evaluated. To that end, we gathered mitochondrial and nuclear data for 230 specimens attributed to the genus Eumunida, including type specimens, for a large proportion of the described species. We also compared the distribution of morphological characters used in the identification keys over the identified genetic clusters. The inclusion of type specimens in the dataset unambiguously links genetic clusters to taxon names.

\section{Materials and methods}

\section{Material and DNA sequencing}

From the collections of the Museum National d'Histoire Naturelle, Paris (MNHN) we selected 230 specimens of Eumunida from the South West Pacific and Indian Oceans (Table 1). Among them, nine are holotypes and 24 are paratypes, representing 13 different species. The 197 remaining specimens were morphologically identified to species level and attributed to 17 valid names of eumunid species. Thus, more than half of the species diversity currently recognised in the genus Eumunida is represented in our dataset (Tables 2, 3). These 17 species hypotheses are represented by $1-95$ specimens, with an average of 12.05 specimens per species
(Table 1). These morphological identifications were used as primary species hypotheses. The morphological characters used in species identification for all the species in the genus were listed and used to build a morphological matrix (Tables 2, 3).

DNA was extracted from a piece of muscle tissue using the DNeasy $^{\circledR} 96$ Tissue kit (Qiagen), and specimens were kept as vouchers. Fragments of the Cytochrome Oxydase I (COI) mitochondrial gene and $28 \mathrm{~S}$ rDNA nuclear gene were amplified using universal primers LCO1490 (5'GGTCAACAAATCATAAAGATATTGG-3') and HCO2198 (5'-TAAACTTCAGGGTGACCAAAAAATCA-3') (Folmer et al. 1994), and $\mathrm{Cl}^{\prime}$ (5'- ACCCGCTGAATTTAAGCAT-3' (Jovelin and Justine 2001) and D2 (5'TCCGTGTTTCAAGACGG-3' (Dayrat et al. 2001). All PCR reactions were performed in $25 \mu \mathrm{L}$, containing $3 \mathrm{ng}$ of DNA, $1 \mathrm{X}$ reaction buffer, $2.5 \mathrm{mM} \mathrm{MgCl}_{2}, 0.26 \mathrm{mM}$ dNTP, $0.3 \mu \mathrm{M}$ of each primer, 5\% DMSO and 1.5 units of Q-Bio Taq, QBiogene for COI gene and Taq Core Kit 2, QBiogene for 28S rDNA gene. Thermocycles consisted of an initial denaturation step at $94^{\circ} \mathrm{C}$ for $4 \mathrm{~min}$, followed by 30 cycles of denaturation at $94^{\circ} \mathrm{C}$ for $30 \mathrm{~s}$, annealing at $48^{\circ} \mathrm{C}$ for $\mathrm{COI}$ gene and $56^{\circ} \mathrm{C}$ for $28 \mathrm{~S}$ rDNA gene for $40 \mathrm{~s}$ and extension at $72^{\circ} \mathrm{C}$ for $1 \mathrm{~min}$. The final extension was at $72^{\circ} \mathrm{C}$ for $10 \mathrm{~min}$. Some PCR products were purified using Montage $\mathrm{T}^{\mathrm{TM}}$ PCR Centrifugal Filter Devices (Millipore) and sequenced on a CEqn $2000^{\mathrm{TM}}$ automated sequencer (Beckman) - corresponding to GenBank accession numbers AY800009-800046, AY800048, AY800050, AY800051, AY800055-800065 and DQ011181-011220. The other PCR products were purified and sequenced by the Genoscope (GenBank accession numbers EU243337-EU243562 for COI gene and EU243574-EU243663 for 28S rDNA gene). In all cases, both directions were sequenced to confirm accuracy of each haplotype sequences.

\section{Phylogenetic analyses}

Sequences were manually aligned for the COI gene, and the Clustal W algorithm (default parameters) implemented in BioEdit (Hall 1999) was used for alignment of our 28S rDNA sequences. Since all the species analysed here belong to a single genus, the sequence variability and the number of gaps for the $28 \mathrm{~S}$ gene were reduced. Consequently, we considered that homology was confidently inferred using Bioedit. The RNAalifold webserver (http://rna.tbi.univie.ac.at/cgi-bin/RNAalifold.cgi) was used to predict a consensus secondary structure for the $28 \mathrm{~S}$ gene and to identify the loops and stems. Loops generally correspond to variable regions, as opposed to stems, which are generally more conserved. In consequence, two different models of evolution were used for the phylogenetic analyses of the $28 \mathrm{~S}$ data. Best-fit models of evolution were selected for the COI genes and for the loops and stems partitions of the 28S gene using Modellgenerator V.85 (Keane et al. 2006) under the Bayesian Information Criterion, with four discrete gamma categories. The best-fit models of evolution are the $\mathrm{HKY}+\mathrm{I}+\mathrm{G}$ (with $\mathrm{I}=0.6$ and $\alpha=0.62)$ for the COI gene, the $\operatorname{TrNef}+\mathrm{I}+\mathrm{G}(\mathrm{I}=0.31, \alpha=0.15)$ for the $28 \mathrm{~S}$ gene, the $\mathrm{K} 80+\mathrm{G}(\alpha=0.5)$ for the loops of the $28 \mathrm{~S}$ gene and the $\mathrm{K} 80+\mathrm{G}(\alpha=0.25)$ for the stems of the $28 \mathrm{~S}$ gene.

As distances-based methods are classically used in barcode studies, a genetic distance matrix including all sequences was 
Table 1. Description of the specimens analysed in this study

\begin{tabular}{|c|c|c|c|c|c|c|}
\hline MNHN ID & Geographic area & Morphological ID & Status & GenBank COI & GenBank 28S & BOLD ID \\
\hline IU-2008-13009 & Norfolk Ridge & sternomaculata & holotype & EU243561 & EU243662 & EUMU225-7 \\
\hline IU-2008-13010 & Norfolk ridge & annulosa & holotype & EU243515 & EU243646 & EUMU179-7 \\
\hline IU-2008-13627 & Norfolk ridge, Brachiopode & sternomaculata & & EU243484 & EU243635 & EUMU148-7 \\
\hline IU-2008-13628 & Norfolk ridge, Kaimon Maru & annulosa & & EU243507 & EU243644 & EUMU171-7 \\
\hline IU-2008-13642 & Norfolk ridge, Introuvable & sternomaculata & & EU243481 & EU243633 & EUMU145-7 \\
\hline IU-2008-13736 & Norfolk ridge, Kaimon Maru & annulosa & & EU243506 & EU243643 & EUMU170-7 \\
\hline IU-2008-13747 & Norfolk ridge, Jumeau est & annulosa & & EU243469 & EU243623 & EUMU133-7 \\
\hline IU-2008-13748 & Norfolk ridge, Jumeau est & annulosa & & EU243470 & $>$ & EUMU134-7 \\
\hline IU-2008-13749 & Norfolk ridge, Eponge & annulosa & & EU243467 & EU243621 & EUMU131-7 \\
\hline IU-2008-13750 & Norfolk ridge, Eponge & annulosa & & EU243468 & EU243622 & EUMU132-7 \\
\hline IU-2008-13751 & Norfolk ridge, Crypthelia & annulosa & & EU243460 & & EUMU124-7 \\
\hline IU-2008-13752 & Norfolk ridge, Crypthelia & annulosa & & EU243461 & EU243618 & EUMU125-7 \\
\hline IU-2008-13753 & Norfolk ridge, Crypthelia & annulosa & & EU243462 & & EUMU126-7 \\
\hline IU-2008-13754 & Norfolk ridge, Crypthelia & annulosa & & EU243463 & EU243619 & EUMU127-7 \\
\hline IU-2008-13755 & Norfolk ridge, Crypthelia & annulosa & & EU243464 & & EUMU128-7 \\
\hline IU-2008-13756 & Norfolk ridge, Crypthelia & annulosa & & EU243465 & & EUMU129-7 \\
\hline IU-2008-13757 & Norfolk ridge, Crypthelia & annulosa & & EU243466 & EU243620 & EUMU130-7 \\
\hline IU-2008-13758 & Norfolk ridge, Introuvable & annulosa & & EU243474 & EU243626 & EUMU138-7 \\
\hline IU-2008-13759 & Norfolk ridge, Introuvable & annulosa & & EU243473 & EU243625 & EUMU137-7 \\
\hline IU-2008-13760 & Norfolk ridge, Stylaster & annulosa & & EU243471 & EU243624 & EUMU135-7 \\
\hline IU-2008-13761 & Norfolk ridge, Stylaster & annulosa & & EU243472 & & EUMU136-7 \\
\hline IU-2008-13762 & Norfolk ridge, Jumeau ouest & annulosa & & EU243489 & & EUMU153-7 \\
\hline IU-2008-13763 & Norfolk ridge, Jumeau ouest & annulosa & & EU243490 & EU243637 & EUMU154-7 \\
\hline IU-2008-13764 & Norfolk ridge, Jumeau ouest & annulosa & & EU243491 & & EUMU155-7 \\
\hline IU-2008-13765 & Norfolk ridge, Jumeau ouest & annulosa & & EU243492 & & EUMU156-7 \\
\hline IU-2008-13766 & Norfolk ridge, Jumeau ouest & annulosa & & EU243493 & EU243638 & EUMU157-7 \\
\hline IU-2008-13767 & Norfolk ridge, Jumeau ouest & annulosa & & EU243494 & EU243639 & EUMU158-7 \\
\hline IU-2008-13768 & Norfolk ridge, Jumeau est & sternomaculata & & EU243456 & EU243615 & EUMU120-7 \\
\hline IU-2008-13769 & Norfolk ridge, Jumeau est & sternomaculata & & EU243457 & & EUMU121-7 \\
\hline IU-2008-13770 & Norfolk ridge, Jumeau est & sternomaculata & & EU243458 & EU243616 & EUMU122-7 \\
\hline IU-2008-13771 & Norfolk ridge, Jumeau est & sternomaculata & & EU243459 & EU243617 & EUMU123-7 \\
\hline IU-2008-13772 & Norfolk ridge, Brachiopode & annulosa & & EU243435 & & EUMU099-7 \\
\hline IU-2008-13773 & Norfolk ridge, Brachiopode & annulosa & & EU243436 & & EUMU100-7 \\
\hline IU-2008-13775 & Norfolk ridge, Antigonia & annulosa & & EU243443 & & EUMU107-7 \\
\hline IU-2008-13776 & Norfolk ridge, Antigonia & annulosa & & EU243444 & & EUMU108-7 \\
\hline IU-2008-13777 & Norfolk ridge, Antigonia & annulosa & & EU243445 & & EUMU109-7 \\
\hline IU-2008-13778 & Norfolk ridge, Crypthelia & annulosa & & EU243447 & & EUMU111-7 \\
\hline IU-2008-13779 & Norfolk ridge, Munida & annulosa & & EU243448 & & EUMU112-7 \\
\hline IU-2008-13780 & Norfolk ridge, Munida & annulosa & & EU243449 & EU243612 & EUMU113-7 \\
\hline IU-2008-13781 & Norfolk ridge, Munida & sternomaculata & & EU243450 & & EUMU114-7 \\
\hline IU-2008-13782 & Island of Pines & annulosa & & EU243451 & EU243614 & EUMU115-7 \\
\hline IU-2008-13785 & Norfolk ridge, Jumeau est & spinosa & & EU243533 & EU243655 & EUMU197-7 \\
\hline IU-2008-13786 & Norfolk ridge, Jumeau est & spinosa & & EU243534 & EU243656 & EUMU198-7 \\
\hline IU-2008-13787 & Norfolk ridge, Jumeau est & spinosa & & EU243535 & & EUMU199-7 \\
\hline IU-2008-13788 & Norfolk ridge, Jumeau est & spinosa & & EU243536 & EU243657 & EUMU200-7 \\
\hline IU-2008-13789 & Norfolk ridge, Jumeau est & spinosa & & EU243537 & & EUMU201-7 \\
\hline IU-2008-13790 & Norfolk ridge, Jumeau est & spinosa & & EU243538 & & EUMU202-7 \\
\hline IU-2008-13791 & Norfolk ridge, Jumeau est & spinosa & & EU243539 & & EUMU203-7 \\
\hline IU-2008-13792 & Norfolk ridge, Jumeau est & spinosa & & EU243540 & & EUMU204-7 \\
\hline IU-2008-13793 & Norfolk ridge, Jumeau est & spinosa & & EU243541 & & EUMU205-7 \\
\hline IU-2008-13794 & Norfolk ridge, Jumeau est & spinosa & & EU243542 & & EUMU206-7 \\
\hline IU-2008-13795 & Solomon Islands & laevimana & & EU243508 & & EUMU172-7 \\
\hline IU-2008-13796 & Solomon Islands & laevimana & & EU243509 & & EUMU173-7 \\
\hline IU-2008-13797 & Guadeloupe & picta & & EU243556 & EU243661 & EUMU220-7 \\
\hline IU-2008-13798 & Guadeloupe & picta & & EU243557 & & EUMU221-7 \\
\hline IU-2008-13799* & Guadeloupe & picta & & EU243558 & & EUMU222-7 \\
\hline IU-2008-13801 & Norfolk ridge, Jumeau est & sternomaculata & & EU243455 & & EUMU119-7 \\
\hline IU-2008-13803 & Norfolk ridge, Jumeau est & sternomaculata & & EU243452 & & EUMU116-7 \\
\hline IU-2008-13804 & Norfolk ridge, Jumeau est & sternomaculata & & EU243453 & & EUMU117-7 \\
\hline
\end{tabular}


Table 1. (continued)

\begin{tabular}{|c|c|c|c|c|c|c|}
\hline MNHN ID & Geographic area & Morphological ID & Status & GenBank COI & GenBank 28S & BOLD ID \\
\hline IU-2008-13806 & Norfolk ridge, Stylaster & sternomaculata & & EU243400 & & EUMU064-7 \\
\hline IU-2011-5396 & Norfolk ridge, Jumeau Ouest & annulosa & & EU243365 & & EUMU029-7 \\
\hline IU-2011-5397 & Norfolk ridge, Eponge & annulosa & & EU243370 & & EUMU034-7 \\
\hline IU-2011-5398 & Norfolk ridge, Eponge & annulosa & & EU243371 & & EUMU035-7 \\
\hline IU-2011-5399 & Norfolk ridge, Eponge & annulosa & & EU243372 & & EUMU036-7 \\
\hline IU-2011-5401 & Norfolk ridge, Eponge & annulosa & & EU243413 & EU243598 & EUMU077-7 \\
\hline IU-2011-5402 & Norfolk ridge, Eponge & annulosa & & EU243414 & EU243599 & EUMU078-7 \\
\hline IU-2011-5403 & Norfolk ridge, Eponge & annulosa & & EU243415 & & EUMU079-7 \\
\hline IU-2011-5404 & Norfolk ridge, Eponge & annulosa & & EU243416 & & EUMU080-7 \\
\hline IU-2011-5405 & Norfolk ridge, Eponge & annulosa & & EU243417 & & EUMU081-7 \\
\hline IU-2011-5406 & Norfolk ridge, Jumeau Ouest & annulosa & & EU243366 & EU243585 & EUMU030-7 \\
\hline IU-2011-5412 & Norfolk ridge, Stylaster & sternomaculata & & EU243478 & EU243630 & EUMU142-7 \\
\hline IU-2011-5413 & Norfolk ridge, Stylaster & sternomaculata & & EU243480 & EU243632 & EUMU144-7 \\
\hline IU-2011-5414 & Norfolk ridge, Stylaster & sternomaculata & & EU243479 & EU243631 & EUMU143-7 \\
\hline IU-2011-5415 & Norfolk ridge, Brachiopode & sternomaculata & & EU243482 & EU243634 & EUMU146-7 \\
\hline IU-2011-5416 & Norfolk ridge, Brachiopode & sternomaculata & & EU243483 & & EUMU147-7 \\
\hline IU-2011-5417 & Norfolk ridge, Brachiopode & sternomaculata & & EU243485 & & EUMU149-7 \\
\hline IU-2011-5418 & Norfolk ridge, Brachiopode & sternomaculata & & EU243486 & & EUMU150-7 \\
\hline IU-2011-5419 & Norfolk ridge, Brachiopode & sternomaculata & & EU243487 & & EUMU151-7 \\
\hline IU-2011-5420 & Norfolk ridge, Brachiopode & sternomaculata & & EU243488 & EU243636 & EUMU152-7 \\
\hline IU-2011-5421 & Norfolk ridge & sternomaculata & paratype & & EU243651 & \\
\hline IU-2011-5422 & Norfolk ridge & sternomaculata & paratype & & EU243652 & \\
\hline IU-2011-5423 & Tuamotu & keijii & & EU243337 & & EUMU001-7 \\
\hline IU-2011-5432 & New-Caledonia, Surprise & parva & & EU243346 & & EUMU010-7 \\
\hline IU-2011-5433 & Norfolk Ridge, Jumeau Est & karubar & & EU243347 & EU243574 & EUMU011-7 \\
\hline IU-2011-5434 & Norfolk Ridge, Jumeau Est & karubar & & EU243348 & & EUMU012-7 \\
\hline IU-2011-5435 & Norfolk Ridge, Jumeau Est & karubar & & EU243349 & EU243575 & EUMU013-7 \\
\hline IU-2011-5436 & Indonesia, Tanimbar and Kai Islands & smithii & & EU243350 & & EUMU014-7 \\
\hline IU-2011-5437 & Indonesia, Tanimbar and Kai Islands & smithii & & EU243351 & & EUMU015-7 \\
\hline IU-2011-5438 & $<<<0$ & treguieri & & EU243352 & EU243576 & EUMU016-7 \\
\hline IU-2011-5439 & Norfolk ridge, Introuvable & annulosa & & EU243353 & EU243577 & EUMU017-7 \\
\hline IU-2011-5440 & Norfolk ridge, Introuvable & annulosa & & EU243354 & EU243578 & EUMU018-7 \\
\hline IU-2011-5441 & Norfolk ridge, Introuvable & sternomaculata & & EU243355 & & EUMU019-7 \\
\hline IU-2011-5442 & Norfolk ridge, Introuvable & sternomaculata & & EU243356 & EU243579 & EUMU020-7 \\
\hline IU-2011-5443 & Norfolk ridge, Introuvable & annulosa & & EU243357 & EU243580 & EUMU021-7 \\
\hline IU-2011-5444 & Polynesia, Raivavae & treguieri & paratype & EU243358 & EU243581 & EUMU022-7 \\
\hline IU-2011-5445 & Tuamotu, Mururoa & treguieri & paratype & EU243359 & EU243582 & EUMU023-7 \\
\hline IU-2011-5446 & Norfolk ridge, Introuvable & annulosa & & EU243360 & EU243583 & EUMU024-7 \\
\hline IU-2011-5447 & Norfolk ridge, Stylaster & annulosa & & EU243361 & EU243584 & EUMU025-7 \\
\hline IU-2011-5448 & Norfolk ridge, Stylaster & annulosa & & EU243362 & & EUMU026-7 \\
\hline IU-2011-5449 & Norfolk ridge, Stylaster & annulosa & & EU243363 & & EUMU027-7 \\
\hline IU-2011-5450 & Norfolk ridge, Stylaster & annulosa & & EU243364 & & EUMU028-7 \\
\hline IU-2011-5451 & Norfolk ridge, Eponge & annulosa & & EU243369 & & EUMU033-7 \\
\hline
\end{tabular}


Table 1. (continued)

\begin{tabular}{|c|c|c|c|c|c|c|}
\hline MNHN ID & Geographic area & Morphological ID & Status & GenBank COI & GenBank 28S & BOLD ID \\
\hline IU-2011-5452 & Norfolk ridge, Introuvable & annulosa & & EU243373 & & EUMU037-7 \\
\hline IU-2011-5453 & Norfolk ridge, Introuvable & annulosa & & EU243374 & & EUMU038-7 \\
\hline IU-2011-5454 & Norfolk ridge, Introuvable & annulosa & & EU243375 & & EUMU039-7 \\
\hline IU-2011-5455 & Norfolk ridge, Introuvable & annulosa & & EU243376 & & EUMU040-7 \\
\hline IU-2011-5456 & Norfolk ridge, Jumeau Est & annulosa & & EU243377 & & EUMU041-7 \\
\hline IU-2011-5457 & Norfolk ridge, Jumeau Est & annulosa & & EU243378 & & EUMU042-7 \\
\hline IU-2011-5458 & Norfolk ridge, Jumeau Est & annulosa & & EU243379 & & EUMU043-7 \\
\hline IU-2011-5459 & Norfolk ridge, Jumeau Est & annulosa & & EU243380 & $>$ & EUMU044-7 \\
\hline IU-2011-5460 & Norfolk ridge, Mont $\mathrm{n}^{\circ} 2$ & annulosa & & EU243381 & & EUMU045-7 \\
\hline IU-2011-5461 & Norfolk ridge, Mont n ${ }^{\circ} 2$ & annulosa & & EU243382 & & EUMU046-7 \\
\hline IU-2011-5462 & Norfolk ridge, Mont $\mathrm{n}^{\circ} 2$ & sternomaculata & & EU243384 & EU243587 & EUMU048-7 \\
\hline IU-2011-5463 & Norfolk ridge, Mont $n^{\circ} 1$ & sternomaculata & & EU243385 & EU243588 & EUMU049-7 \\
\hline IU-2011-5464 & Norfolk ridge, Mont $n^{\circ} 1$ & sternomaculata & & EU243386 & EU243589 & EUMU050-7 \\
\hline IU-2011-5465 & Norfolk ridge, Mont $\mathrm{n}^{\circ} 1$ & sternomaculata & & EU243387 & & EUMU051-7 \\
\hline IU-2011-5466 & Norfolk ridge, Mont $n^{\circ} 1$ & sternomaculata & & EU243388 & EU243590 & EUMU052-7 \\
\hline IU-2011-5467 & Norfolk ridge, Stylaster & sternomaculata & & EU243389 & & EUMU053-7 \\
\hline IU-2011-5468 & Norfolk ridge, Stylaster & sternomaculata & & EU243390 & & EUMU054-7 \\
\hline IU-2011-5469 & Norfolk ridge, Stylaster & sternomaculata & & EU243391 & & EUMU055-7 \\
\hline IU-2011-5470 & Norfolk ridge, Stylaster & sternomaculata & & EU243392 & & EUMU056-7 \\
\hline IU-2011-5471 & Norfolk ridge, Eponge & sternomaculata & & EU243393 & & EUMU057-7 \\
\hline IU-2011-5472 & Norfolk ridge, Eponge & sternomaculata & & EU243394 & & EUMU058-7 \\
\hline IU-2011-5473 & Norfolk ridge, Eponge & sternomaculata & & EU243395 & & EUMU059-7 \\
\hline IU-2011-5474 & Norfolk ridge, Introuvable & sternomaculata & & EU243396 & EU243591 & EUMU060-7 \\
\hline IU-2011-5475 & Norfolk ridge, Introuvable & sternomaculata & & EU243397 & & EUMU061-7 \\
\hline IU-2011-5476 & Norfolk ridge, Introuvable & sternomaculata & & EU243398 & & EUMU062-7 \\
\hline IU-2011-5477 & Norfolk ridge, Introuvable & sternomaculata & & EU243399 & & EUMU063-7 \\
\hline IU-2011-5478 & Norfolk ridge, Stylaster & annulosa & & EU243401 & & EUMU065-7 \\
\hline IU-2011-5479 & Norfolk ridge, Stylaster & annulosa & & EU243402 & EU243592 & EUMU066-7 \\
\hline IU-2011-5480 & Norfolk ridge, Stylaster & annulosa & & EU243403 & & EUMU067-7 \\
\hline IU-2011-5481 & Norfolk ridge, Stylaster & annulosa & & EU243404 & EU243593 & EUMU068-7 \\
\hline IU-2011-5482 & Norfolk ridge, Stylaster & annulosa & & EU243405 & & EUMU069-7 \\
\hline IU-2011-5483 & Norfolk ridge, Stylaster & annulosa & & EU243406 & & EUMU070-7 \\
\hline IU-2011-5484 & Norfolk ridge, Introuvable & annulosa & & EU243407 & EU243594 & EUMU071-7 \\
\hline IU-2011-5485 & Norfolk ridge, Introuvable & annulosa & & EU243408 & & EUMU072-7 \\
\hline IU-2011-5486 & Norfolk ridge, Introuvable & annulosa & & EU243409 & & EUMU073-7 \\
\hline IU-2011-5487 & Norfolk ridge, Introuvable & annulosa & & EU243410 & EU243595 & EUMU074-7 \\
\hline IU-2011-5488 & Norfolk ridge, Introuvable & annulosa & & EU243411 & EU243596 & EUMU075-7 \\
\hline IU-2011-5489 & Norfolk ridge, Jumeau Est & annulosa & & EU243418 & EU243600 & EUMU082-7 \\
\hline IU-2011-5490 & Norfolk ridge, Jumeau Est & annulosa & & EU243419 & & EUMU083-7 \\
\hline IU-2011-5491 & Norfolk ridge, Jumeau Est & annulosa & & EU243420 & EU243601 & EUMU084-7 \\
\hline IU-2011-5492 & Norfolk ridge, Jumeau Est & annulosa & & EU243421 & & EUMU085-7 \\
\hline IU-2011-5493 & Norfolk ridge, Jumeau Est & annulosa & & EU243422 & EU243602 & EUMU086-7 \\
\hline IU-2011-5494 & Norfolk ridge, Stylaster & sternomaculata & & EU243423 & EU243603 & EUMU087-7 \\
\hline IU-2011-5495 & Norfolk ridge, Stylaster & sternomaculata & & EU243424 & & EUMU088-7 \\
\hline IU-2011-5496 & Norfolk ridge, Stylaster & sternomaculata & & EU243425 & EU243604 & EUMU089-7 \\
\hline IU-2011-5497 & Norfolk ridge, Stylaster & sternomaculata & & EU243426 & & EUMU090-7 \\
\hline IU-2011-5498 & Norfolk ridge, Stylaster & sternomaculata & & EU243427 & EU243605 & EUMU091-7 \\
\hline IU-2011-5499 & Norfolk ridge, Stylaster & sternomaculata & & EU243428 & & EUMU092-7 \\
\hline IU-2011-5500 & Norfolk ridge, Eponge & sternomaculata & & EU243429 & EU243606 & EUMU093-7 \\
\hline IU-2011-5501 & Norfolk ridge, Eponge & sternomaculata & & EU243430 & & EUMU094-7 \\
\hline IU-2011-5502 & Norfolk ridge, Eponge & sternomaculata & & EU243431 & & EUMU095-7 \\
\hline IU-2011-5503 & Norfolk ridge, Eponge & sternomaculata & & EU243432 & EU243607 & EUMU096-7 \\
\hline IU-2011-5504 & Norfolk ridge, Eponge & sternomaculata & & EU243433 & & EUMU097-7 \\
\hline IU-2011-5505 & Norfolk ridge, Eponge & sternomaculata & & EU243434 & EU243608 & EUMU098-7 \\
\hline IU-2011-5506 & Norfolk ridge, Brachiopode & annulosa & & EU243437 & EU243609 & EUMU101-7 \\
\hline IU-2011-5507 & Norfolk ridge, Brachiopode & annulosa & & EU243438 & EU243610 & EUMU102-7 \\
\hline IU-2011-5508 & Norfolk ridge, Kaimon Maru & annulosa & & EU243439 & & EUMU103-7 \\
\hline IU-2011-5509 & Norfolk ridge, Kaimon Maru & annulosa & & EU243440 & & EUMU104-7 \\
\hline IU-2011-5510 & Norfolk ridge, Kaimon Maru & annulosa & & EU243441 & & EUMU105-7 \\
\hline
\end{tabular}


Table 1. (continued)

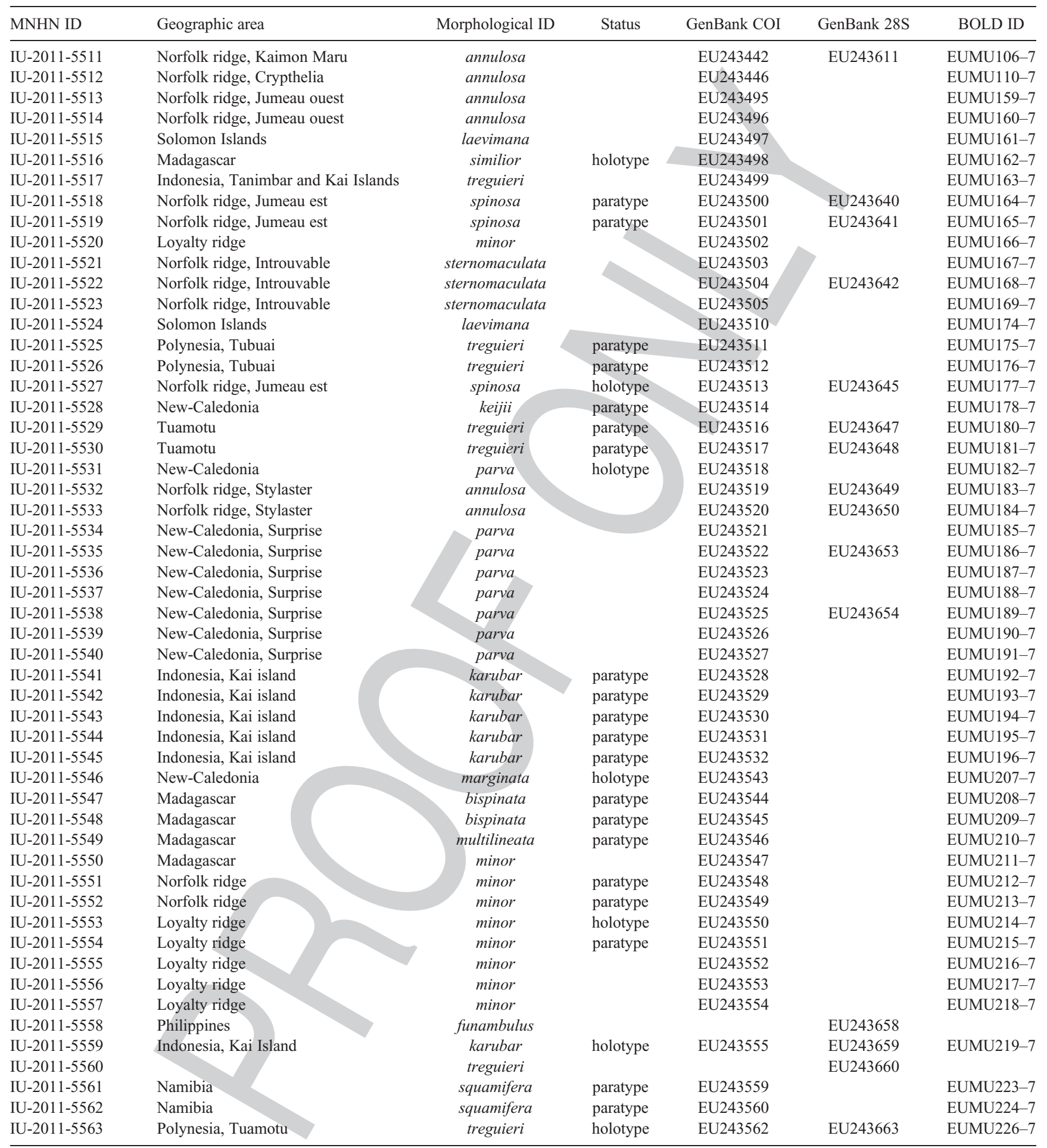

calculated for the COI gene under the K2P model and used to reconstruct a Neighbour-Joining tree, using MEGA 5 (Tamura et al. 2011). To accurately reconstruct the phylogenetic relationships within Eumunida, a Bayesian Analysis was also conducted using Mr. Bayes (Huelsenbeck et al. 2001); it consisted of two independent analyses (six Markov chains,
30000000 generations, with a sampling frequency of one tree each 5000 generations). One different model (each with 6 substitution categories, a gamma-distributed rate variation across sites approximated in four discrete categories and a proportion of invariable sites) was applied for each partition (COI, 28S loops and 28S stems). Convergence of each 
Table 2. Description of morphological characters

\begin{tabular}{|c|c|c|}
\hline & Characters & States \\
\hline 1 & Thoracic spines & $\mathrm{Yes}=1, \mathrm{No}=0$ \\
\hline 2 & Posterior region of carapace with complete striae & $\mathrm{Yes}=1, \mathrm{No}=0$ \\
\hline 3 & Number of anterolateral spines on each side & One spine $=1$, two spines $=0$ \\
\hline 4 & Pad on palm of cheliped & Yes $=1, \mathrm{No}=0$ \\
\hline 5 & Epigastric spines & Yes $=1, \mathrm{No}=0$ \\
\hline 6 & Posterior part of abdominal tergites, after last stria, smooth & $\mathrm{Yes}=1, \mathrm{No}=0$ \\
\hline 7 & Depressed area on branchial region of carapace & $\mathrm{Yes}=1, \mathrm{No}=0$ \\
\hline 8 & Mesiodorsal row of spines on cheliped palm & $\mathrm{Yes}=1, \mathrm{No}=0$ \\
\hline 9 & First anterolateral spine less than half lateral supraorbital & Yes $=1$ (less), No $=0$ (more) \\
\hline 10 & Distal spines on carpus of chelipeds & 2 spp. $=1,3$ spp. $=0$ \\
\hline 11 & Distal spine on merus of third maxilliped & $\mathrm{Yes}=1, \mathrm{No}=0$ \\
\hline 12 & Male pleopods & $\mathrm{Yes}=1, \mathrm{No}=0$ \\
\hline 13 & Six to seven spines on upper margin of propodus walking leg & $\mathrm{Yes}=1, \mathrm{No}=0$ \\
\hline 14 & Row of ventral spines on merus of chelipeds & $5-8$ spp. $=1,1$ sp. $=0$ \\
\hline 15 & Ocular peduncles short, not reaching end of lateral supraorbital spines & $\mathrm{Yes}=1, \mathrm{No}=0$ \\
\hline 16 & Lateral surface of 4 th pereiopod with spine & Yes $=1, \mathrm{No}=0$ \\
\hline
\end{tabular}

Table 3. Character state for each species of Eumunida

Species for which molecular data were obtained are shown in bold. See Table 2 for explanation of the morphological characters used

\begin{tabular}{|c|c|c|c|c|c|c|c|c|c|c|c|c|c|c|c|c|}
\hline & 1 & 2 & 3 & 4 & 5 & 6 & 7 & 8 & 9 & 10 & 11 & 12 & 13 & 14 & 15 & 16 \\
\hline E. ampliata & 0 & 1 & 1 & 1 & 0 & 1 & 0 & 0 & 1 & 0 & 0 & 0 & 0 & 1 & 0 & 1 \\
\hline E. annulosa & 1 & 1 & 0 & 1 & 0 & 1 & 0 & 1 & 1 & 1 & 0 & 0 & 0 & 0 & 0 & 1 \\
\hline E. australis & 1 & 1 & 0 & 1 & 0 & 1 & 0 & 0 & 0 & 0 & 0 & 0 & 0 & 1 & 1 & 1 \\
\hline E. balssi & 0 & 1 & 0 & 0 & 0 & 1 & 0 & 1 & 1 & 0 & 1 & 0 & 0 & 0 & 0 & 1 \\
\hline E. bella & 1 & 1 & 1 & 1 & 0 & 1 & 0 & 1 & 0 & 0 & 0 & 0 & 0 & 0 & 0 & 1 \\
\hline E. bispinata & 0 & 1 & 0 & 0 & 0 & 0 & 0 & 0 & 1 & 1 & 0 & 0 & 0 & 0 & 0 & 1 \\
\hline E. capillata & $\bar{\equiv}$ & 1 & 0 & 1 & 0 & 0 & 0 & 0 & 1 & 0 & 0 & 0 & 0 & 0 & 0 & 0 \\
\hline \multicolumn{17}{|l|}{ E. chani } \\
\hline E. debilistriata & 0 & 0 & 1 & 0 & 0 & 0 & 0 & 1 & 0 & 0 & 0 & 0 & 1 & 1 & 0 & 1 \\
\hline E. depressa & 1 & 1 & 1 & 1 & 0 & 0 & 1 & 0 & 0 & 0 & 0 & 0 & 0 & 1 & 0 & 0 \\
\hline E. dofleini & 0 & 0 & 1 & 0 & 0 & 0 & 0 & 1 & 0 & 0 & 0 & 0 & 0 & 0 & 0 & 0 \\
\hline E. funambulus & 1 & 1 & 1 & 1 & 1 & 0 & 0 & 1 & 0 & 0 & 1 & 0 & 1 & 1 & 0 & 1 \\
\hline E. gordonae & 0 & 0 & 0 & 1 & 1 & 0 & 0 & 0 & 1 & 0 & 0 & 0 & 0 & 0 & 0 & 0 \\
\hline E. karubar & 0 & 1 & 0 & 0 & 0 & 0 & 0 & 1 & 1 & 0 & 0 & 1 & 0 & 1 & 0 & 1 \\
\hline E. keijii & 1 & 1 & 1 & 0 & 0 & 0 & 0 & 1 & 0 & 0 & 0 & 0 & 0 & 0 & 0 & 1 \\
\hline E. laevimana & 0 & 1 & 1 & 0 & 0 & 0 & 0 & 0 & 0 & 1 & 0 & 0 & 0 & 1 & 0 & 0 \\
\hline E. macphersoni & 1 & 1 & 1 & 0 & 0 & 0 & 0 & 1 & 1 & 0 & 0 & 0 & 0 & 0 & 1 & 0 \\
\hline E. marginata & 0 & 1 & 0 & 1 & 1 & 0 & 0 & 1 & 1 & 0 & 1 & 0 & 0 & 0 & 0 & 0 \\
\hline E. minor & 0 & 1 & 0 & 1 & 0 & 0 & 0 & 0 & 1 & 0 & 1 & 0 & 0 & 1 & 0 & 1 \\
\hline E. multilineata & 1 & 0 & 1 & 1 & 0 & 0 & 0 & 1 & 1 & 0 & 0 & 0 & 0 & 1 & 0 & 0 \\
\hline E. pacifica & 1 & 1 & 1 & 0 & 0 & 0 & 0 & 1 & 0 & 0 & 0 & 0 & 0 & 0 & 0 & 1 \\
\hline E. parva & 0 & 1 & 0 & 0 & 0 & 0 & 0 & 0 & 1 & 0 & 0 & 1 & 0 & 0 & 0 & 1 \\
\hline E. picta & 1 & 1 & 0 & 1 & 0 & 0 & 0 & 0 & 0 & 0 & 0 & 0 & 0 & 0 & 0 & 1 \\
\hline E. similior & 1 & 1 & 1 & 1 & 0 & 1 & 0 & 0 & 0 & 0 & 0 & 0 & 0 & 1 & 0 & 0 \\
\hline E. smithii & 0 & 1 & 0 & 0 & 0 & 0 & 0 & 1 & 1 & 0 & 0 & 1 & 0 & 1 & 1 & 1 \\
\hline E. spinosa & 0 & 1 & 0 & 0 & 0 & 0 & 0 & 0 & 0 & 0 & 0 & 0 & 0 & 0 & 0 & 1 \\
\hline E. squamifera & 1 & 0 & 1 & 1 & 0 & 0 & 0 & 0 & 0 & 0 & 0 & 0 & 0 & 0 & 0 & 1 \\
\hline E. sternomaculata & 1 & 1 & 0 & 1 & 0 & 0 & 0 & 1 & 0 & 0 & 0 & 0 & 0 & 0 & 0 & 1 \\
\hline E. treguieri & 1 & 1 & 1 & 1 & 0 & 0 & 0 & 0 & 0 & 0 & 0 & 0 & 0 & 0 & 1 & 1 \\
\hline Eumunida sp. nov. & 1 & 1 & 0 & 1 & 0 & 0 & 0 & 1 & 0 & 0 & 0 & 0 & 0 & 0 & 0 & 1 \\
\hline
\end{tabular}

analysis was evaluated using Tracer 1.4.1 (Rambaut and Drummond 2007), and analyses were terminated when ESS values were all greater than 200. We also used the AWTY application (a system for graphical exploration of Markov
Chain Monte Carlo convergence in Bayesian phylogenetic inference) for each run (two runs for the COI genes and two for the $28 \mathrm{~S}$ gene): the cumulative split frequencies were stable after the burn-in phase, the split frequencies in run pairs 
('compare' analysis) were strongly correlated and the betweenrun distance was included in the range of the within-run distances for more than half of the generations ( 'var' analysis). A consensus tree was then calculated after omitting the first $25 \%$ trees as burnin. For both genes, we used Munida acantha (Macpherson, 1994) as an outgroup to artificially root the tree (GenBank accession numbers: AY800033 for COI gene and EU249347 for 28S rDNA gene).

\section{Results}

\section{Mitochondrial dataset}

We obtained 226 COI sequences of 658 bp in length with 219 polymorphic sites corresponding mostly to the first (47) and third (164) codon position. This dataset is available in the BOLD project 'Eumunida barcodes and taxonomy' under the accession numbers EUMU001-07 to EUMU226-07. The maximum K2P distance between pairs of COI sequences of the genus Eumunida is 0.158 , with a minimum of 0 and a mean of 0.079 (Fig. $1 A$ ). The histogram representing all the distances between types and nontype specimens defines two groups (Fig. 1A): the first, with an upper boundary of 0.033 , includes all the distances between two type specimens of one species, but also distances between the holotype of E. parva (de Saint Laurent \& Macpherson, 1990) and the type specimens (one holotype and five paratypes) of E. karubar (de Saint Laurent \& Poupin, 1996); the second, characterised by a lower boundary of 0.043 , includes only interspecific comparisons between types. Neighbour-Joining and Bayesian phylogenetic trees were highly congurent (only the Bayesian tree is shown in Fig. 2A) and revealed 16 terminal genetic units: genetic distances within each cluster are less than 0.033, and COI sequences placed in different genetic units are separated by genetic distances greater than 0.043 . Among these 16 genetic units, 13 include several specimens and all are highly supported (Posterior Probabilities pp. $=1$ ), and 10 contain one or several type specimens.

\section{Nuclear dataset}

The 28S rDNA gene was much more difficult to sequence, especially for older museum specimens and, as a consequence, fewer specimens were sequenced compared with the $\mathrm{CO} 1$ dataset. We obtained 89 sequences of $867 \mathrm{bp}$. Two groups of K2P distances are separated by a gap on the genetic distances histogram (Fig. 1B). The short-distance group has an upper bound of 0.001 and the long-distance group has a lower bound of 0.018 . For each pair of specimens, a genetic distance less than 0.001 for this dataset corresponds to a genetic distance less than

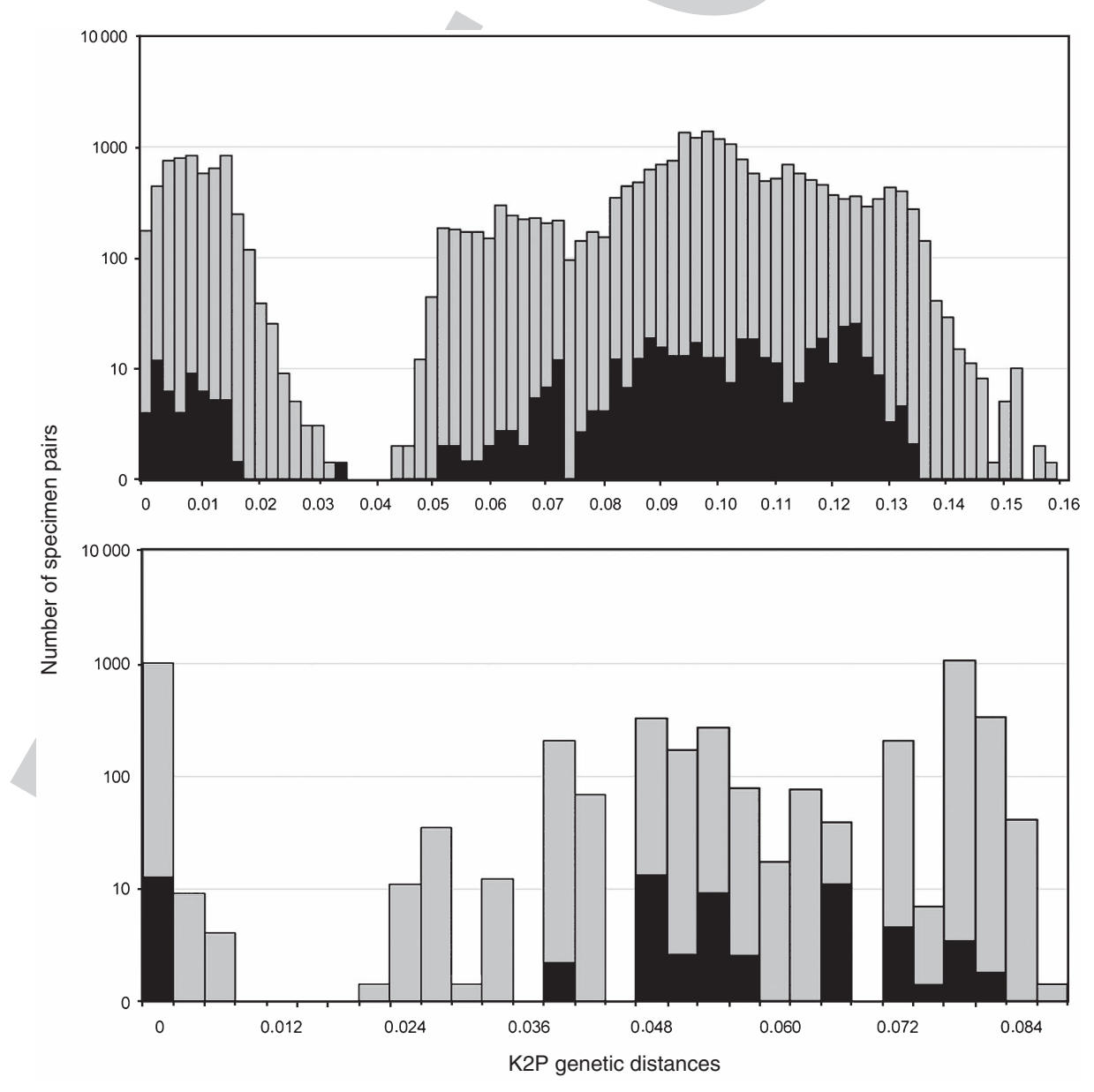

Fig. 1. Histogram of genetic distances for $(A)$ the COI gene and $(B)$ the $28 \mathrm{~S}$ gene datasets. Black bars: pairs of type specimens. Grey bars: pairs of non-type specimens. 
A

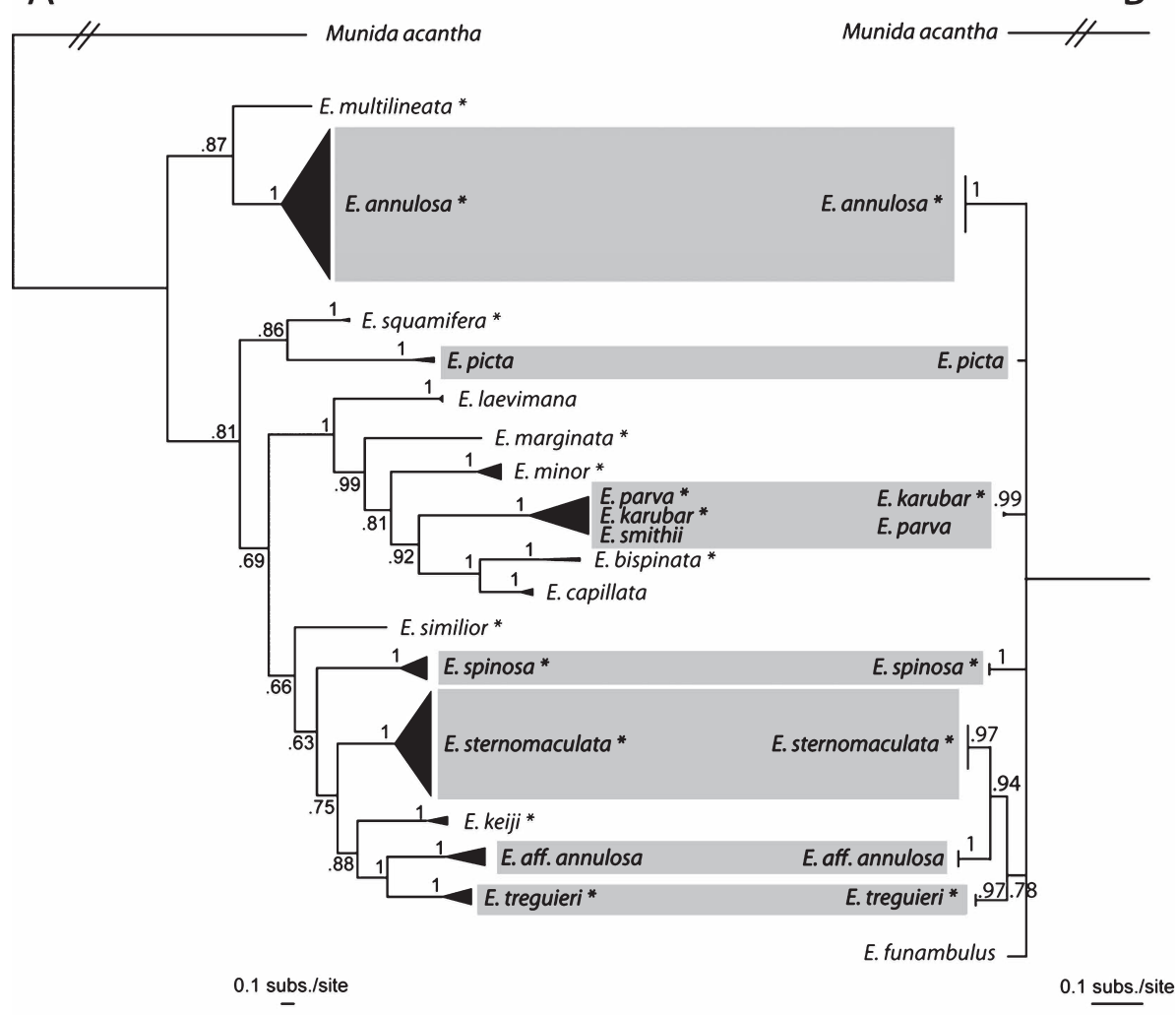

B

subs./site

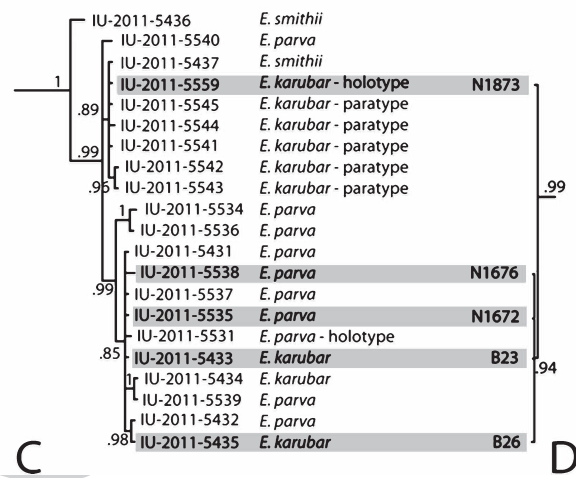

Fig. 2. (A) Bayesian tree for COI gene dataset, with posterior probabilities indicated for each node. Clades are collapsed in triangles, with the height representing the number of specimens and the width the length of the branches. An asterisk indicates units that include a type specimen. $(B)$ Bayesian tree for the $28 \mathrm{~S}$ gene dataset. (C) Detail of the COI gene tree for the E. parva/E. karubar/E. smithii clade. (D) Detail of the $28 \mathrm{~S}$ gene tree for the E. parva/E. karubar/E. smithii clade.

0.033 with the COI gene. Conversely, when the genetic distance between two $28 \mathrm{~S}$ rDNA sequences is greater than 0.018 , the genetic distance between COI sequences corresponding to the same specimens is greater than 0.043 . The intraspecific distances between type specimens fall in the short-distance group whereas interspecific distances between type specimens fall in the longdistance group. The $28 \mathrm{~S}$ dataset reveals the same monophyletic lineages as the COI dataset: among the 16 lineages defined with the COI gene, seven correspond with clusters identified by the $28 \mathrm{~S}$ gene (Fig. 2B). Furthermore, one additional lineage, not sequenced with the COI gene, is defined with the $28 \mathrm{~S}$ gene. The deeper nodes of the $28 \mathrm{~S}$ tree are not as well resolved as the CO1 tree but the terminals are highly supported in all cases.

\section{Genetic units and species names}

On the basis of separate analyses of the two molecular datasets, we are able to define 17 genetically distinct units (Fig. 2) that may be considered as species hypotheses. Eleven of these units include at least one sequence of one type specimen (holotype and/or paratypes) for at least one of the two genes, and can be directly linked to a species name. Types were included for E. annulosa de 
Saint Laurent \& Macpherson, 1990, E. bispinata Baba, 1990, E. keiji de Saint Laurent \& Macpherson, 1990, E. marginata de Saint Laurent \& Macpherson, 1990, E. minor de Saint Laurent \& Macpherson, 1990, E. multilineata de Saint Laurent \& Poupin, 1996, E. similior Baba, 1990, E. spinosa Macpherson, 2006, E. squamifera de Saint Laurent \& Macpherson, 1990, E. sternomaculata de Saint Laurent \& Macpherson, 1990 and E. treguieri de Saint Laurent \& Poupin, 1996. Four other genetic units do not include type specimens but their identification is

Q1 based on morphological identification keys: E. capillata de Saint Laurent \& Macpherson, 1990, E. funambulus Gordon, 1930, E. laevimana Gordon, 1930, and E. picta Smith, 1883. The name ' $E$. annulosa' is attributed to two clades, one including the holotype. Since the specimens of the genetic unit without the holotype look like those from $E$. annulosa but are not closely related to E. annulosa (Fig. $2 A, B$ ), in accordance with the Code of Zoological Nomenclature, we named this genetic group $E$. aff. annulosa. Finally, the remaining genetic unit unites specimens morphologically assigned to three different species (E. karubar de Saint Laurent \& Poupin, 1996; E. parva de Saint Laurent \& Macpherson, 1990, and E. smithii Henderson, 1885). For the COI dataset, the holotype of E. parva, five paratypes of E. karubar and the holotype of E. karubar are included within the same genetic unit (Fig. 2C). Genetic distances between sequences of paratypes and/or holotypes falling into this well supported clade are lower than between other paratypes of a single species name placed in a single clade (e.g. the two paratypes of E. bispinata).

\section{Discussion}

The barcoding gap

In our analysis, the distribution pattern of genetic distances for the two gene fragments used allows us to cluster genetically similar individuals that are separated from each other by relatively large distances. In the bimodal distribution of distances, the lower bound of the first mode - small distances - and the upper bound of the second mode-large distances (Meier et al.2008) - are reliably estimated thanks to the larger number of specimens analysed, allowing the assertion that the observed gap is not an artefact resulting from a sampling bias. We are fully aware, like others (e.g. Meyer and Paulay 2005; Costa et al. 2007; Hajibabaei et al. 2007; Wiemers and Fiedler 2007; Meier et al. 2008), of the importance of the sampling scheme to interpret a gap in the distribution of the pairwise genetic distances, but insist that the originality of our dataset is the inclusion of type specimens. Interestingly, all the genetic distances between the paratypes of a given name fall in the first mode whereas genetic distances among the holotypes (and the paratypes from different names) fall into the second mode (except for the type specimens of E. karubar and E. parva), suggesting that the gap may be used in a first approach as a species threshold.

\section{Concordance of most genetic units with primary species hypotheses}

Inclusion of a closely related outgroup in the analysis shows that each of the 17 defined genetic units has it own evolutionary history. Moreover, the two gene trees obtained with our two unlinked genetic markers are in concordance. This concordance suggests that genetic exchanges among individuals from different clades are unlikely. A previous study has shown that in two of these genetic units, gene flow occurs between populations over the geographic range of each species but not between species (Samadi et al. 2006). These 17 genetic units can thus be considered robust species hypotheses.

Among them, 15 units cluster specimens attributed to a unique species and a single name using the morphological identification key. Ten of these 15 species clusters also include type specimens. These 15 clusters are therefore delimited unambiguously, even though inclusion of type specimens in such genetic units is the only way to unambiguously attribute species names to them; but even though five units do not include the type specimen for the name attributed from the key, we can define 15 primary species hypotheses as the best ones given the available data to date. However, our result is not fully congruent with previous species hypotheses, of which four are questioned by the molecular analysis. Indeed, our data suggest (1) the occurrence of a cryptic species (i.e. not yet identified using morphology) that needs a new name because no type specimen can be attributed to the corresponding cluster, and (2) the grouping of three previously admitted species hypotheses into one, and thus the synonymy of three available species names.

\section{A cryptic species under the name E. annulosa}

The genetic divergence found between $E$. annulosa and $E$. aff. annulosa largely exceeds the average divergence found not only within the other species hypotheses of our dataset, but also within other galatheoid species (Machordom and Macpherson 2004). Since one of the two clades includes the holotype of E. annulosa, the other clade ( $E$. aff. annulosa), not yet detected by morphologists, should indisputably be described under a new name (Fig. 2A, B). Although this clade is more closely related to $E$. treguieri in the tree, the morphological characters differ only slightly from those of E. annulosa or E. sternomaculata. These two species are distinguished morphologically by the relative length of the first pair of anterolateral spines (longer in E. sternomaculata than in E. annulosa), the presence of two (E. annulosa) or three (E. sternomaculata) distal spines on the carpus of the chelipeds, and the posterior part of the abdominal tergites, after last stria (smoother in E. annulosa than in E. sternomaculata) (Table 3: Characters 6, 9 and 10). The larger specimens of $E$. aff. annulosa display intermediate states for two characters: the relative size of the first anterolateral spine is intermediate between that described for E. annulosa and that described for E. sternomaculata and a $3 \mathrm{rd}$ distal spine is present on the cheliped carpus, but is generally very small. However, these morphological characters, on which this new species may be diagnosed, are difficult to observe on small specimens and thus are useful only for identification of adult specimens. Since the two species are morphologically very close but do not display sister relationships, they are 'cryptic species', and not 'sibling species', as defined by Bickford et al. (2007). This result stresses the importance of molecular analyses to detect such 'cryptic species', not only within this genus but also in other crustacean decapods (see 
the review by Knowlton 2000 and Bickford et al. 2007). Contrary to most studies, which provide (at best) molecular data for name-bearing specimens of new species names (e.g. Shih et al. 2010; Ahyong et al. 2010), the inclusion of many name-bearing specimens in the analysis points to the necessity of a new name for this 'cryptic species'. For such cryptic taxa, the DNA barcode is obviously a more effective identification tool than a morphological identification key, being informative at all life stages and thus having broader applications (e.g. De Ley et al. 2005; Savolainen et al. 2005; Vences et al. 2005).

\section{Synonymy of E. karubar, E. parva and E. smithii}

Our analysis also suggests that three named species hypotheses (E. karubar, E. parva, E. smithii) should actually be merged into a single species hypothesis. When using a morphological identification key, the specimens attributed to each one of these three species names, including the five paratypes, the holotype of E. karubar and the holotype of E. parva, are scattered among the different subclades without showing any obvious significant pattern (Fig. 2C,D).

The morphological distinction among E. parva, E. karubar and E. smithii is based on the occurrence (E. smithii and E. karubar) or absence (E. parva) of ventral spines on the merus of the chelipeds and on the presence (E. smithii and E. karubar) or absence (E. parva) of some ventromesial spines on the palm of the chelipeds (Table 3: Characters 8 and 14). The distinction among these species is also based on the length of the ocular peduncles (shorter in E. smithii than in E. karubar and E. parva) (Saint Laurent and Poupin 1996). By combining data from morphology, geography, and independent genetic characters, we suggest that the three names are synonymous (this amounts to considering E. parva and E. karubar as junior synonyms of $E$. smithii). This interpretation may yet be challenged by the molecular analysis of the holotype of E. smithii. Such an analysis could not be conducted for this study because the type specimens for this name were collected during the Challenger Expedition (1874-76), are not housed at the MNHN, and tissue was not available for sequencing. Consequently, we used topotypic specimens collected from the type locality (Kei Islands, Indonesia). According to our interpretation, the morphological differences upon which description of new species hypotheses bearing new species names has been based in the past are the expression of intraspecific variability. This would imply that variability should be used with caution as a diagnostic trait at species level in this genus. The alternative hypothesis would be recent speciation events leading to low genetic divergence.

Therefore, we propose that the genus Eumunida contains 28 species (see also Baba et al. 2008; Schnabel and Ahyong 2010), including the new cryptic species of E. annulosa and considering $E$. parva and E. karubar as junior synonyms of $E$. smithii). The diagnosis of E. smithii is as follows:

\section{Diagnosis of E. smithii}

Carapace with distinct transverse ridges, laterally armed with 6 spines; 2 spines anterior to posterior cervical groove, anterior spine subequal to posterior spine, about half as long as lateral supraocular spine. No spine on gastric region. Third maxilliped merus with median spine and without distal spine on flexer margin. Sternite 3 with paired median spines; Sternite 4 unarmed on each side. Cheliped carpus with 3 terminal spines; palm without ventral pad of densely packed hairs, longer than fingers, relatively massive, covered with short fine setae. Rudimentary pleopods present on abdominal segments $2-5$ in males.

\section{Name-bearing specimens integrated into a molecular revision of species hypotheses}

One of the main problems when revising species hypotheses and identifying specimens in the context of DNA-barcoding projects is the naming procedure. An appropriate sampling effort within species, a large taxonomic coverage within the genus, and the inclusion of as many type specimens as possible are necessary when confronting morphological species hypotheses to independent characters (DNA polymorphism) and various species delimitation criteria. In the case of the genus Eumunida, it allowed us (1) to support most of the morphology-based primary species hypotheses, (2) to bring up new hypotheses, and (3) to point to the necessity of a taxonomic revision. Overall, although we detected two discrepancies between our data and the current state of the taxonomy of Eumunida, our results suggest that most morphological traits commonly used in this genus to propose primary species hypotheses stand up when other characters are used. By contrast with most studies, the inclusion of name-bearing specimens in the molecular study allows us to correctly assign names to the supported or reformulated species hypotheses and to unquestionably determine whether new names are needed or whether some names should be considered synonyms of older names. This point is particularly critical when cryptic species are detected, i.e. when morphological keys do not help to attribute names to genetic units. Last, even though several Eumunida species are missing in this study and should be barcoded in the future, our study shows that the COI gene fragment is an effective tool to attribute species names to specimens, and vice versa, in Eumunida, which is the primary purpose of DNA barcoding.

\section{Acknowledgements}

We are grateful to Bertrand Richer de Forges, cruise leader of several deep-sea cruises of the Tropical Deep Sea Benthos program on board R/V Alis, that generated the deep-sea samples used in this study. All material has been collected under appropriate collection permits and approved ethics guidelines. This work was supported by the 'Consortium National de Recherche en Génomique', and the 'Service de Systematique Moléculaire' of the Muséum National d'Histoire Naturelle (UMS 2700 CNRS-MNHN). It is part of the agreement $n^{\circ} 2005 / 67$ between the Genoscope and the Muséum National d'Histoire Naturelle on the project 'Macrophylogeny of Life' directed by Guillaume Lecointre. These data fed the MarBol project supported by the Sloan Foundation. We are also pleased to thank Anouk Barberousse, Philippe Bouchet, Mande Holford, Line Le Gall and Simon Tillier for constructive discussions and/or comments on, and English improvements of, the manuscript, and Julien Brisset and Laure Corbari for the curation of the collection. 


\section{References}

Ahyong, S. T., Chan, T.-Y., and Bouchet, P. (2010). Mighty claws: a new genus and species of lobster from the Philippine deep sea (Crustacea, Decapoda, Nephropidae). Zoosystema 32(3), 525-535. doi:10.5252/ z2010n3a11

Baba, K., Macpherson, E., Poore, G. C. B., Ahyong, S. T., Bermudez, A., Cabezas, P., Lin, C. W., Nizinski, M., Rodrigues, C., and Schnabel, K. E. (2008). Catalogue of squat lobsters of the world (Crustacea: Decapoda: Anomura - families Chirostylidae, Galatheidae and Kiwaidae). Zootaxa 1905, 1-220.

Bickford, D., Lohman, D. J., Sodhi, N. S., Ng, P. K. L., Meier, R., Winker, K., Ingram, K. K., and Das, I. (2007). Cryptic species as a window on diversity and conservation. Trends in Ecology \& Evolution 22(3), 148-155. doi:10.1016/j.tree.2006.11.004

Bouchet, P., Héros, V., Lozouet, P., and Maestrati, P. (2008). A quater-century of deep-sea malacological exploration in the South and West Pacific: Where do we stand? How far to go? Mémoires du Muséum national d'Histoire naturelle 196, 9-40.

Costa, F. O., deWaard, J. R., Boutillier, J., Ratsnasingham, S., Dooh, R. T., Hajibabaei, M., and Hebert, P. D. N. (2007). Biological identifications through DNA barcodes: the case of the Crustacea. Canadian Journal of Fisheries and Aquatic Sciences 64, 272-295. doi:10.1139/f07-008

Dayrat, B. (2006). A taxonomic revision of Paradoris sea slugs (Mollusca, Gastropoda, Nudibranchia, Doridina). Zoological Journal of the Linnean Society 147, 125-238. doi:10.1111/j.1096-3642.2006.00219.x

Dayrat, B., Tillier, A., Lecointre, G., and Tillier, S. (2001). New clades of Euthyneuran gastropods (Mollusca) from 28S rRNA sequences. Molecular Phylogenetics and Evolution 19(2), 225-235. doi:10.1006/ mpev.2001.0926

De Ley, P., Tandingam De Ley, I. T., Morris, K., Abebe, E., Mundo-Ocampo, M., Yoder, M., Heras, J., Waumann, D., Rocha-Olivares, A., Jay Burr, A. H., Baldwin, J. G., and Thomas, W. K. (2005). An integrated approach to fast and informative morphological vouchering of nematodes for applications in molecular barcoding. Philosophical Transactions of the Royal Society B 360, 1945-1958. doi:10.1098/rstb.2005.1726

Folmer, O., Black, M., Hoeh, W., Lutz, R., and Vrijenhoek, R. (1994). DNA primers for amplification of mitochondrial cytochrome coxidase subunit I from diverse metazoan invertebrates. Molecular Marine Biology and Biotechnology 3, 294-299.

Hajibabaei, M., Singer, G. A. C., Hebert, P. D. N., and Hickey, D. A. (2007). DNA barcoding: how it complements taxonomy, molecular phylogenetics and population genetics. Trends in Genetics 23, 167-172. doi:10.1016/j. tig.2007.02.001

Hall, T. A. (1999). BioEdit: a user-friendly biological sequence alignment editor and analysis program for Windows 95/98/NT. Nucleic Acids Symposium Series 41, 95-98.

Hebert, P. D. N., and Gregory, T. R. (2005). The promise of DNA Barcoding for taxonomy. Systematic Biology 54, 852-859. doi:10.1080/ 10635150500354886

Huelsenbeck, J. P., Ronquist, F., and Hall, B. (2001). MrBayes: Bayesian inference of phylogeny. Bioinformatics (Oxford, England) 17, 754-755. doi:10.1093/bioinformatics/17.8.754

Jovelin, R., and Justine, J.-L. (2001). Phylogenetic relationships within the Polyopisthocotylean monogeneans (Plathyhelminthes) inferred from partial 28S rDNA sequences. International Journal for Parasitology 31(4), 393-401. doi:10.1016/S0020-7519(01)00114-X

Keane, T. M., Creevey, C. J., Pentony, M. M., Naughton, T. J., and McInerney, J. O. (2006). Assessment of methods for amino acid matrix selection and their use on empirical data shows that $a d$ hoc assumptions for choice of matrix are not justified. BMC Evolutionary Biology 6, 29. doi:10.1186/ 1471-2148-6-29
Knowlton, N. (2000). Molecular genetic analyses of species boundaries in the sea. Hydrobiologia 420, 73-90. doi:10.1023/A:1003933603879

Machordom, A., and Macpherson, E. (2004). Rapid radiation and cryptic speciation in galatheid crabs of the genus Munida and related genera in the South West Pacific: molecular and morphological evidence. Molecular Phylogenetics and Evolution 33, 259-279. doi:10.1016/j. ympev.2004.06.001

Meier, R., Zhang, G., and Ali, F. (2008). The use of mean instead of smallest interspecific distances exaggerates the size of the "Barcoding Gap" and leads to misidentification. Systematic Biology 57(5), 809-813. doi: $10.1080 / 10635150802406343$

Meyer, C. P., and Paulay, G. (2005). DNA Barcoding: error rates based on comprehensive sampling. PLoS Biology 3(12), e422. doi:10.1371/ journal.pbio.0030422

Padial, J. M., and De La Riva, I. (2007). Integrative taxonomists should use and produce DNA barcodes. Zootaxa 1586, 67-68.

Rambaut, A., and Drummond, A. J. (2007). Tracer v1.4. Available from http:// beast.bio.ed.ac.uk/Tracer

Saint Laurent, M. d., and Poupin, J. (1996). Crustacea, Anomura: Les espèces indo-ouest pacifiques du genre Eumunida Smith, 1880 (Chirostylidae). Description de six espèces nouvelles. In 'Résultats des Campagnes MUSORSTOM, volume 15. Vol. 168'. (Ed. A Crosnier.) pp. 337-385. (Mémoires du Museum National d'Histoire Naturelle: Paris.)

Samadi, S., Bottan, L., McPherson, E., Richer De Forges, B., and Boisselier, M. C. (2006). Seamount endemism questioned by the geographic distribution and population genetic structure of marine invertebrates. Marine Biology 149, 1463-1475. doi:10.1007/s00227-006-0306-4

Savolainen, V., Cowan, R. S., Vogler, A. P., Roderick, G. K., and Lane, R. (2005). Towards writing the encyclopedia of life: an introduction to DNA barcoding. Philosophical Transactions of the Royal Society B 360, 1805-1811. doi:10.1098/rstb.2005.1730

Schindel, D. E., and Miller, S. E. (2005). DNA barcoding a useful tool for taxonomists. Nature 435, 17. doi:10.1038/435017b

Schnabel, K. E., and Ahyong, S. T. (2010). A new classification of the Chirostyloidea (Crustacea: Decapoda: Anomura). Zootaxa 2687, 56-64.

Shih, H.-T., Naruse, T., and Ng, P. K. L. (2010). Uca jocelynae sp. nov. a new species of fiddler crab (Crustacea: Brachyura: Ocypodidae) from the Western Pacific. Zootaxa 2337, 47-62.

Smith, A. M., Wood, D. M., Janzen, D. H., Hallwachs, W., and Hebert, P. D. N (2007). DNA barcodes affirm that 16 species of apparently generalist tropical parasitoid flies (Diptera, Tachinidae) are not all generalists. Proceedings of the National Academy of Sciences of the United States of America 104, 4967-4972. doi:10.1073/pnas.0700050104

Tamura, K., Peterson, D., Peterson, N., Stecher, G., Nei, M., and Kumar, S. (2011). MEGA5: Molecular Evolutionary Genetics Analysis using Q3 Maximum Likelihood, Evolutionary Distance, an $\rightleftharpoons$ ximum Parsimony methods. Molecular Biology and Evolution $\overline{\bar{\Sigma}} 0.1093 /$ molbev/msr121

Vences, M., Thomas, M., Bonett, R. M., and Vieites, D. R. (2005). Deciphering amphibian diversity through DNA barcoding: chances and challenges. Philosophical Transactions of the Royal Society B 360(1462), 1859-1868. doi:10.1098/rstb.2005.1717

Wiemers, M., and Fiedler, K. (2007). Does the DNA barcoding gap exist? - a case study in blue butterflies (Lepidoptera: Lycaenidae). Frontiers in Zoology 4, 8. doi:10.1186/1742-9994-4-8 


\section{AUTHOR QUERIES}

1. Author: 'Four other genetic units do not include type specimens but their identification is based on...' - is rewording $\mathrm{OK}$ ?

2. Author: what does the asterisk denote in Table 1?

3. Author: Please provide volume and page range for reference, "Tamura et al. (2011)". 